\title{
A NOVEL BENZOCYCLOBUTENE-BASED DEVICE FOR STUDYING THE PHYSICS OF THE EBULLITION PROCESS
}

\author{
Saeed Moghaddam ${ }^{1}$, Kenneth T. Kiger ${ }^{1}$, Alireza Modafe ${ }^{2}$, and Reza Ghodssi ${ }^{2}$ \\ ${ }^{1}$ Department of Mechanical Engineering \\ ${ }^{2}$ MEMS Sensors and Actuators Lab (MSAL) \\ Department of Electrical and Computer Engineering, Institute for Systems Research \\ University of Maryland, College Park, MD USA
}

\begin{abstract}
We report, for the first time, simultaneous measurement of temperature and heat flux with a resolution of about $35 \mu \mathrm{m}$ underneath/around a bubble. This was achieved by fabrication of a multilayer Silicon/Benzocyclobutene (BCB) structure with embedded temperature sensors. The sensors are radially distributed around a cavity, with energy input by a thin film heater microfabricated on the backside of the membrane structure. Single bubbles were generated from the cavity while the temperatures and the bubble images were recorded with a sampling frequency of $8 \mathrm{kHz}$. Analysis of the results revealed unique details of the nucleation dynamics and its associated heat transfer processes.
\end{abstract}

\section{INTRODUCTION}

Boiling heat transfer has been considered one of the most efficient mechanisms of heat transfer, and as such, has been implemented in wide variety of applications ranging from nuclear reactors to electronic cooling. Over the past 50 years, scientists have developed several competing mechanistic models to describe the boiling heat transfer process. Although the developed models are intended to predict the heat transfer coefficient at macroscales, their fundamental assumptions lie on complex microscale sub-processes that remain to be experimentally verified. Two main unresolved issues regarding these sub-processes are: 1) dynamics of bubble growth and associated heat transfer processes and 2) the bubble's role in surface heat transfer during the boiling process.

Although it is common knowledge that bubble growth in heterogeneous boiling is due to liquid evaporation at the bubble/liquid interface, the mechanism of heat transfer during this process is yet unknown. The two main competing views on the mechanism of heat transfer to a bubble are typified by the models of Mikic and Rohsenow [1] and Cooper [2]. Mikic and Rohsenow [1] suggested that energy transfer into a bubble predominately comes from the superheated liquid that covers the bubble dome. In contrast, Cooper [2] suggested that evaporation of a thin liquid layer (the so called microlayer) underneath the bubble (i.e. at the bubble/surface contact area) is the main contributing factor into the bubble growth. Numerous studies (e.g. Mei et al. [3] and Robinson and Judd [4]) have been conducted to evaluate the accuracy of the two proposed models. Unfortunately, the lack of experimental data at microscales, where the phenomenon takes place, hasn't allowed a full examination/verification of the two models and their fundamental assumptions.
The second unresolved issue in the boiling process is how a bubble affects the heat transfer around the nucleation site, or in a broader sense how the individual bubbles contribute to the net energy flux from the heated surface. In general, boiling models can be classified into two following categories: 1) single phase convection models and 2) combined single phase convection and latent heat transfer models. The first group of models suggests that surface heat transfer is mainly due to liquid motion induced by bubbles. The exact nature of this convection process is still unknown. Different analogies have suggested convection at macro- and micro-scales. The macroscale models suggest that heat transfer to the liquid is the result of local agitation generated by the wake of departing bubbles from the heat transfer surface [5]. The micro-scale models suggest that bubbles act as micropumps and continuously pump the hot liquid away from the surface [6]. The second group of studies (e.g. Judd et al. [7]) accounts for latent heat transfer from the surface along with the single-phase convection. To the best of our knowledge, the accuracy of the fundamental assumptions of these heat transfer models hasn't been experimentally verified.

Motivated by a need to develop an advanced two-phase microcooler for electronics thermal management, we have conducted a comprehensive study to understand the nucleation dynamics and mechanism of heat transfer during the boiling process. This was achieved through fabrication of a composite wall with embedded Resistance Temperature Detectors (RTDs) that can measure the surface temperature and heat flux distribution near the nucleation site. The device consists of an array of temperature sensors embedded in an $10.5-\mu \mathrm{m}$ thick multilayer Benzocyclobutene (BCB) structure on a $60-\mu \mathrm{m}$ thick, $3.6 \times 3.6 \mathrm{~mm}^{2}$ square-shape silicon membrane. The $60-\mu \mathrm{m}$ thick silicon membrane was designed to keep the lower surface of the BCB structure at a constant temperature level during the bubbling process. BCB was selected among several other lowconductivity polymers such as SU-8, polyimide, and PDMS, because of its microfabrication properties and its stability at high temperatures. The thickness of the $\mathrm{BCB}$ layers and the sensor geometry were determined in a design trade off between the heat flux measurement accuracy and interference with the nucleation process.

The surface is heated by a thin film resistor microfabricated on the backside of the membrane. Bubbles are generated at a dedicated nucleation site at the center of the sensor array. The surface temperature and heat flux underneath/around a bubble along with the images of the bubbles were recorded at a frequency of $8 \mathrm{kHz}$. Results provided the necessary data to explain nucleation dynamics and 
different mechanisms of heat transfer involved in the boiling process. This paper describes the fabrication and testing of the device.

\section{FABRICATION PROCESS}

The device was fabricated on a $300-\mu \mathrm{m}$ thick n-type $<100>$ silicon wafer with silicon oxide and silicon nitride layers on both sides. The nitride and oxide layers were patterned and etched using the RIE process over a $3.6 \times 3.6 \mathrm{~mm}^{2}$ square-shape area on the backside of the wafer. The silicon was then etched through $\mathrm{KOH}$ anisotropic etching to make a $60-\mu \mathrm{m}$ thick membrane. Temperature sensor $\mathrm{T}_{1}$ (see Figure 1) was fabricated on the silicon nitride layer, followed by a $7.5-\mu \mathrm{m}$ thick BCB layer that was spin-coated and fixed by a soft bake. The $T_{2}$ sensor was then fabricated on the preceding BCB layer, with its sensing element centered and aligned to the $T_{1}$ sensor, but the two sensor's leads rotated to the opposite side. Note that the temperature difference between sensors $T_{1}$ and $T_{2}$ determines the spatially averaged heat flux in the boiling site region. The structure was subsequently covered with a second BCB layer (2.5 $\mu \mathrm{m}$ thick), and the sensor array was then fabricated on the resulting surface. Note that the temperature difference between each element of the sensor array $\left(T_{i}\right)$ and $T_{1}$ determines the spatially resolved heat flux. The sensor array was subsequently covered with a $0.5-\mu \mathrm{m}$ thick BCB layer and was hard baked. The BCB layer over all the bond pads was then etched. Initial trials determined that the $\mathrm{Cr} / \mathrm{Ni} / \mathrm{Au}$ bond pads constructed during the sensor fabrication were not stiff enough to allow direct wire bonding. The original pads were etched and $0.5 \mu \mathrm{m} \mathrm{Ti} / 1.5 \mu \mathrm{m} \mathrm{Al}$ bond pads were fabricated at their place. Figures 2 and 3 show the top view of the device. A $0.3-\mu \mathrm{m}$ thick alumina layer was then deposited on the backside of the membrane and a chromium heater with $\mathrm{Au}$ leads was subsequently fabricated on the alumina layer. In the last fabrication stage, three cavities (shown in Figure 4) were fabricated at the center of the sensor array using Focused Ion Beam (FIB).

\section{EXPERIMENTAL APPARATUS}

The device was attached and wire bonded to a custom made Pin Grid Array (PGA). The PGA was then installed on the bottom cap of a chamber that contains the test liquid. FC-72 (developed by $3 \mathrm{M}$ company) was used for the tests, as it is a strong candidate for electronic cooling applications. The chamber pressure was controlled via a connecting bellows and a pressure regulating system (see Figure 5). A hot water line was connected to the external jacket of the test chamber to control the test liquid temperature. The PGA was connected from below to a custom signal conditioning board, and routed to an $\mathrm{A} / \mathrm{D}$ card installed in a PC. All sensors were sampled with a frequency of $8 \mathrm{kHz}$. Also, a CCD camera, synchronized with the A/D board, was used to capture the bubble images with a rate of 8000 frames/sec.

\section{DATA REDUCTION AND ANALYSIS}

Temperature of the test liquid was set at different levels by adjusting the temperature of the hot water supplied to the chamber jacket. Data was collected at different surface temperatures by varying the power applied to the thin film heater on the backside of the membrane. An example set of data at a liquid temperature of $52.7{ }^{\circ} \mathrm{C}$ under $1 \mathrm{~atm}$ pressure is presented and analyzed in the following.

Figure 6 shows a typical bubbling event with its corresponding temperature data shown in Figure 7. An approximate value for the heat flux could be calculated using $q=k_{B C B}\left(T_{1}-T_{i}\right)$. This assumes, however, essential a perfect material with zero heat capacity. To properly account for transient thermal energy stored in the $\mathrm{BCB}$, a numerical model of the BCB layer was built using Icepak (a numerical software developed by Fluent Corporation) to accurately determine the surface heat flux. The experimental temperature profiles were applied to the model and surface heat flux at the area covered by each sensor was determined. Figure 8 shows the numerically calculated heat flux profiles. The temperature and heat flux results and the images of the bubbles have been used to analyze different aspects of the nucleation dynamics and the boiling heat transfer sub-processes. A detailed interpretation and analysis of the experimental results is far beyond the scope of this paper and will be presented in future publications. Only a brief analysis of the results, limited to the broad overview of the phenomena presented in the introduction section, will be discussed in the following.

As can be seen in Figure 8, formation of a bubble is associated with a sudden spike in surface heat flux at the bubble/surface contact area. This indicates a rapid cool down of the surface due to microlayer evaporation. Comparison of the microlayer evaporation energy with the total bubble energy (calculated using the bubble volume) indicated that microlayer accounts only for $12.8 \%$ of the total energy transferred into the bubble for this particular case. The rest of the bubble energy came from the superheated liquid surrounding the bubble dome. This indicates that neither of the bubble growth models $[1,2]$ accurately represents the actual bubble growth mechanism observed in this study.

The results also revealed the details of the heat transfer process from the surface. As can be seen in Figure 8, after the initial heat flux spike at the contact area caused by microlayer evaporation, the heat flux at the contact area approached to zero due to surface dry-out. This trend continued until the contact line started to recede shortly after it reached a radius of 225 $\mu \mathrm{m}$, near the middle of the S- 5 sensor. The liquid front started to cool the surface in a process commonly known as transient conduction heat transfer mode. Contrary to what has been commonly assumed in classical boiling models, transient conduction mode occurred mainly before, and not after, the bubble departure. Calculating the total heat transfer during this process suggested that transient conduction accounts for $24 \%$ of the total heat transfer from the sensor area. Also, comparison of the heat transferred by the microlayer with the total heat transfer from the sensor area suggested that latent heat transfer (i.e. microlayer evaporation) accounts for about $13 \%$ of the total heat transfer. The rest of the heat transfer from the sensor area $(63 \%)$ occurred outside the contact area. As can be seen in Figure 8 , temperature of this region didn't change during the bubble growth and departure and the steady state heat flux in this region can be reasonably calculated using $q=k_{B C B}\left(T_{1}-T_{i}\right)$. An additional test in natural convection mode (in the absence of the bubbles) showed that although heat flux at this region didn't change with the frequency of the bubbling event, it was approximately 3 times higher than the natural convection heat flux at the same wall temperature. 


\section{CONCLUSIONS}

A novel device for direct measurement of the temperature and heat flux fields around a bubble nucleation site with an unprecedented spatial resolution of $35 \mu \mathrm{m}$ was developed. Capability of the device in revealing some aspects of the processes involved in the boiling heat transfer was demonstrated. Results of this study enhance the understanding of the physics of heat transfer process in boiling that is the building block for development of more efficient microcooling devices for electronics cooling applications.

\section{REFERENCES}

[1] B. B. Mikic and W. M. Rohsenow, "Bubble Growth Rates in Non-Uniform Temperature Field", Prog. Heat Mass Transfer, 2, 283 (1969).

[2] M. G. Cooper, "The Microlayer and Bubble Growth in Nucleate Pool Boiling", Int. J. Heat Mass Transfer, 12, 915 (1969).

[3] M. R. Mei, W. Chen, and J. F. Klausner, "Vapor bubble growth in heterogeneous boiling-I \& II", Int. J. Heat Mass Transfer, 38, 909 (1995).

[4] A. J. Robinson and R. L. Judd, "Bubble Growth in a Uniform and Spatially Distributed Temperature Field", Int. J. Heat Mass Transfer, 44, 2699 (2001).

[5] W. M. Rohsenow, "A Method of Correlating Heat Transfer Data for Surface Boiling of Liquids", ASME J. Heat Transfer, 74, 969 (1951).

[6] H. K. Forster and R. Greif, "Heat Transfer to a Boiling Liquid-Mechanism and Correlations", ASME J. Heat Transfer, 81, 43 (1959).

[7] R. L. Judd and K. S. Hwang, "A Comprehensive Model for Nucleate Pool Boiling Heat Transfer Including Microlayer Evaporation", ASME J. Heat Transfer, 98, 623 (1976).

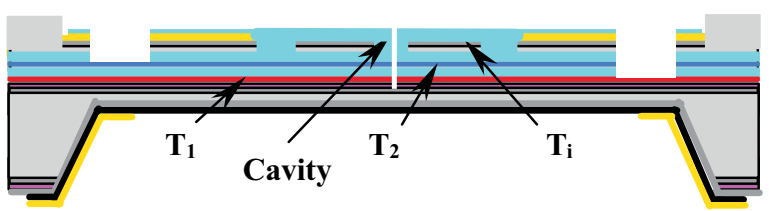

Figure 1. Cross section of the device.

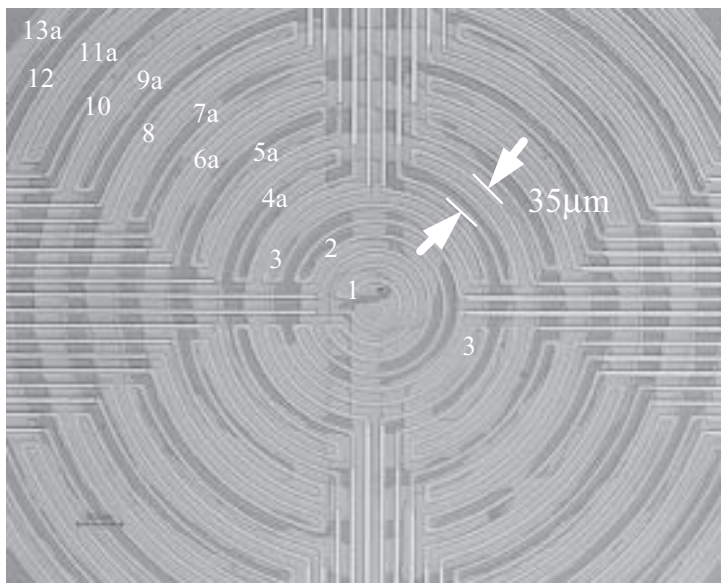

Figure 2. Top view of the device showing sensor array on top of sensors $T_{1}$ and $T_{2}$. Sensor array covers a $1 \mathrm{~mm}$ in diameter circular area

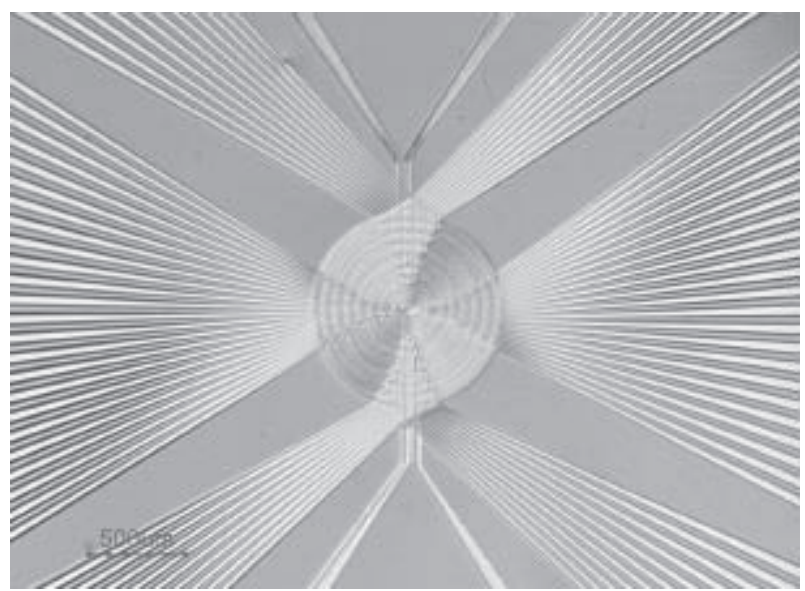

Figure 3. Wide view of the device showing leads of the sensor array and two pairs of leads for sensors $T_{1}$ and $T_{2}$.

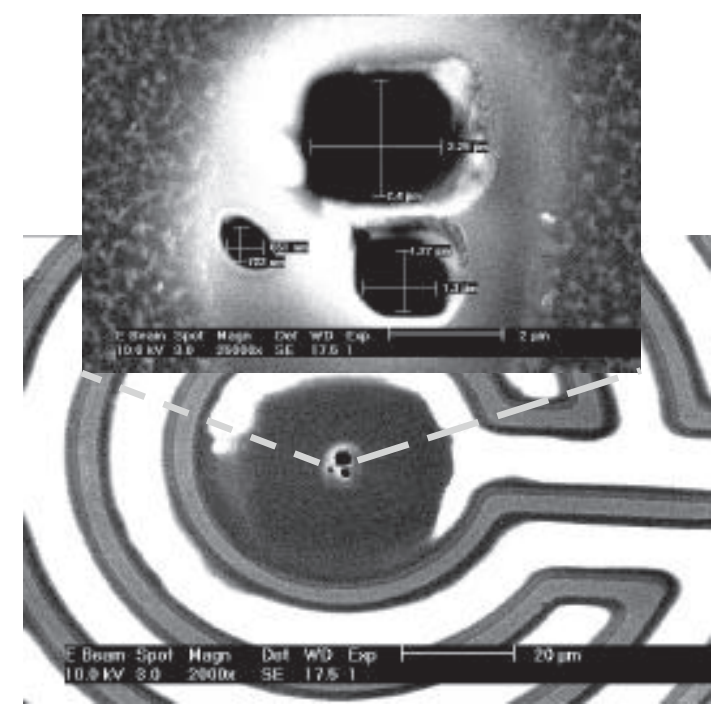

Figure 4. SEM view of the central sensor with cavities about 0.7, 1.3, $2.4 \mu \mathrm{m}$ in diameter at the center. Each cavity is active in a certain temperature range.

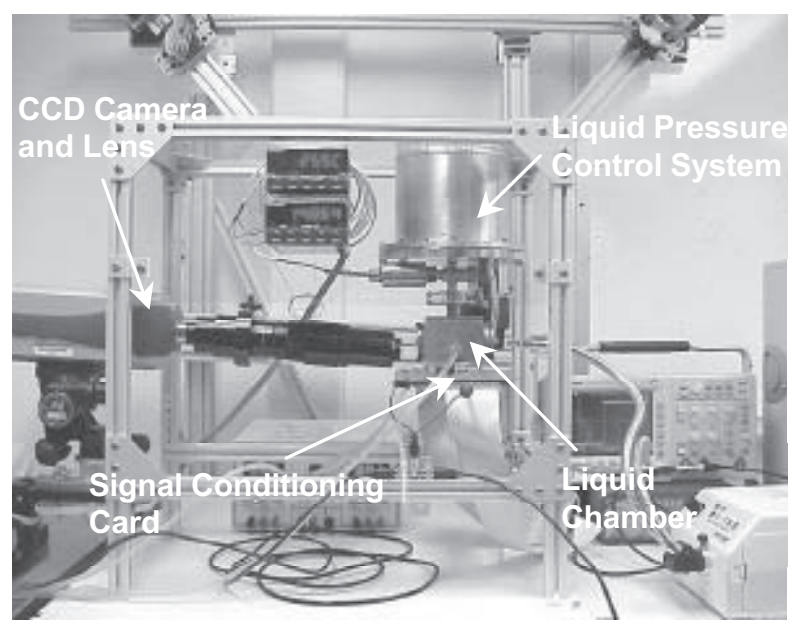

Figure 5. Experimental setup 


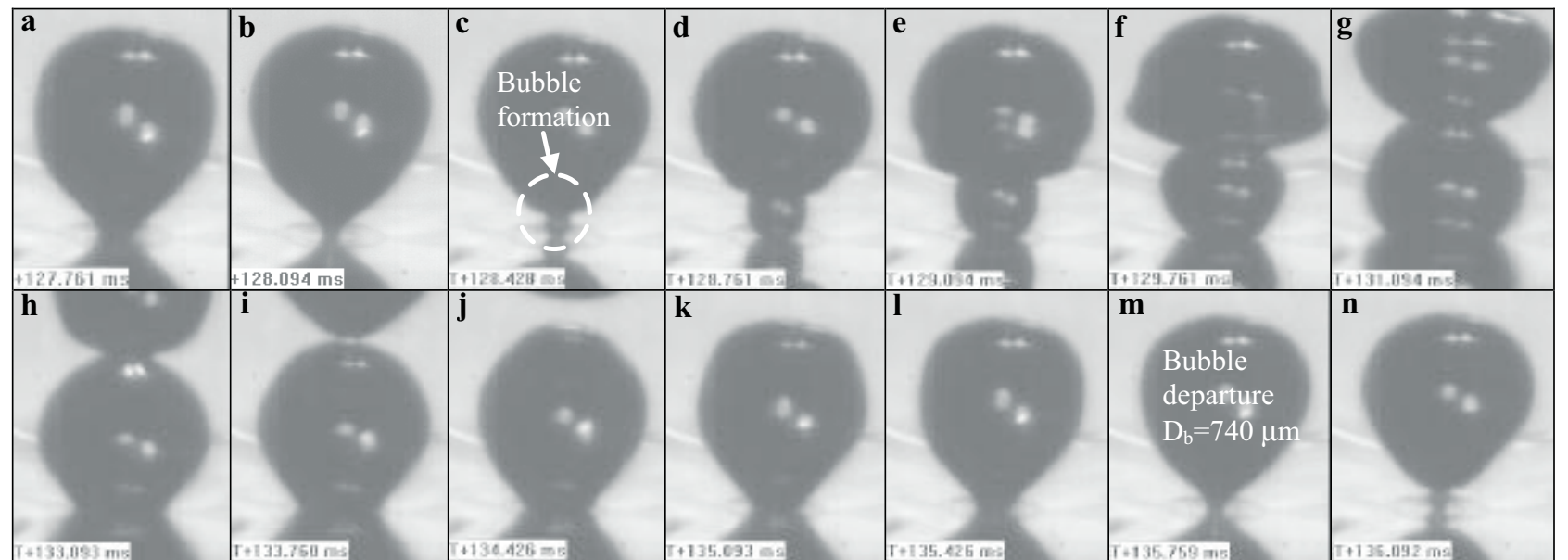

Figure 6. Images of a sample bubbling event. Note that the particular bubble for which the temperature and the heat flux variations are shown in Figures 8\&9, forms at $t=128.428 \mathrm{~ms}$ (image “c”) and departs at $t=135.749 \mathrm{~ms}$ (image “ $\mathrm{m}$ ”).

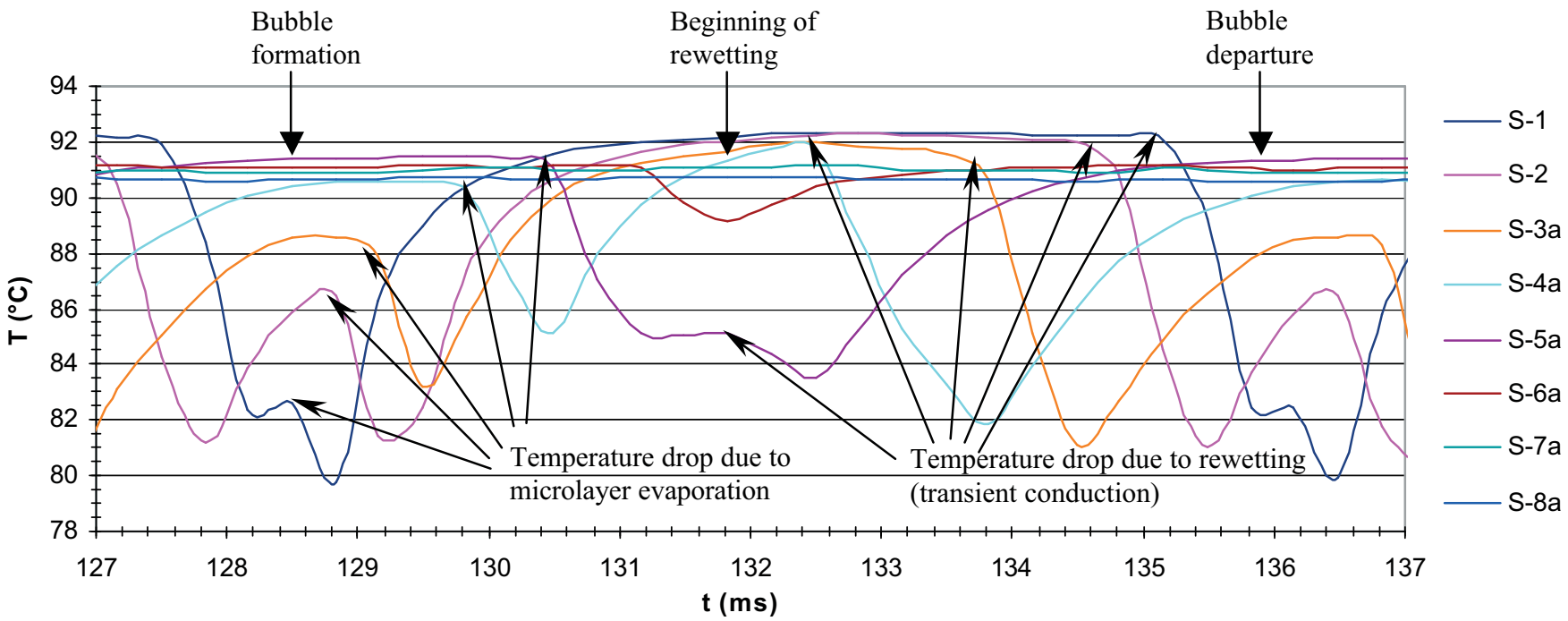

Figure 7. Surface temperature variations under the bubble shown in Figure 7 (see Figure 1 for positions of sensors S-1 to S-8a). Temperature of sensors 9 a to 13 a is equal to sensor 8 a. Temperatures are symmetric at the four sensor array quadrants.

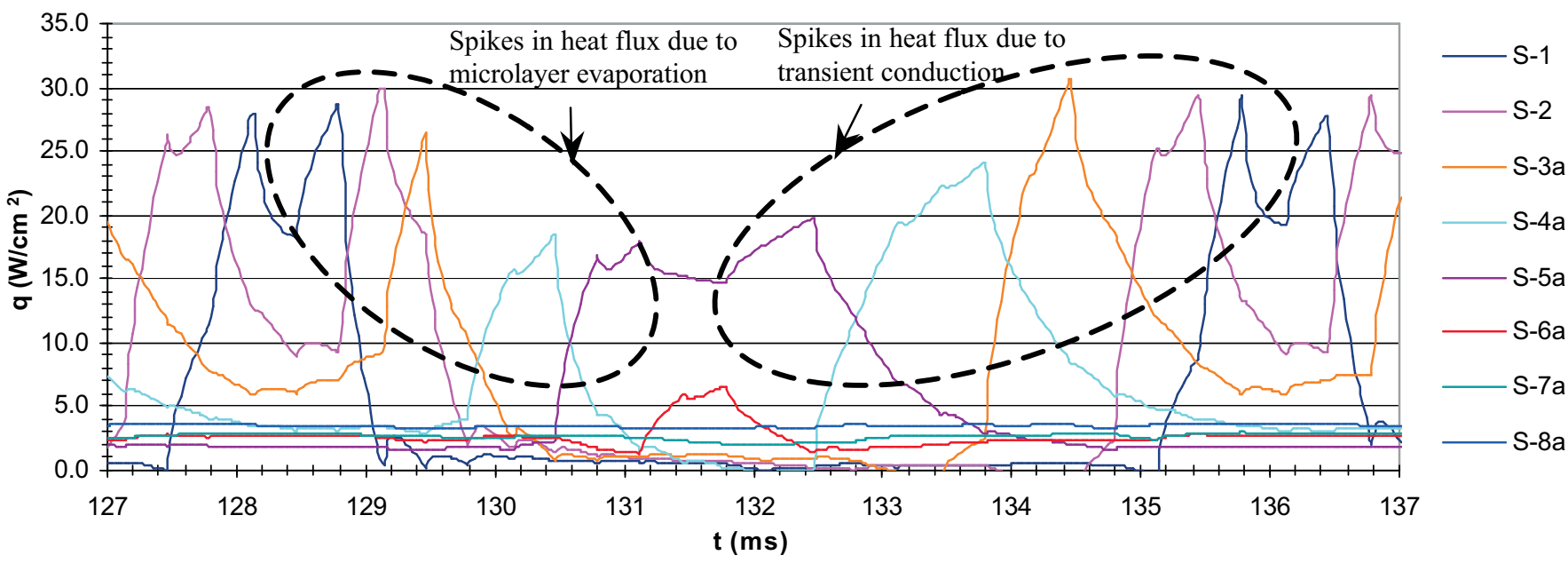

Figure 8. Heat flux variations at the surface for the bubble shown in Figure 7. The area under each curve shows the total heat transfer. These results are numerically modified to account for sensor thermal cross talk and some very fast transient events. 